\title{
Hacia una taxonomía de las prácticas extensionistas en perspectiva histórica
}

\section{On a Taxonomy of Extension Practices [in Historical Perspective]}

\author{
Carlos A. Zavaro Pérez \\ Universidad Nacional de La Plata \\ Argentina \\ czavaro@museo.fcnym.edu.ar
}

Recibido: 4/05/2020 Aceptado: 20/08/2020

Resumen. Se analizan algunas modalidades en las que se ha objetivado históricamente la práctica extensionista, destacando tanto algunas de sus particularidades como el límite de la categoría en la que se inscribe, como la relevancia del contexto económico y sociopolítico en que estas acontecen. Se discuten algunas categorías como paradigma, formato, dispositivo y herramienta, que resultan útiles en su sistematización, constituyendo un sistema taxonómico jerárquico que es definido por dos paradigmas (el paradigma transferencista y el paradigma problematizador) bajo los que pueden aglutinarse los distintos formatos que se reconocen y entre los que se encuentran el formato divulgativo, el formato concientizador, el formato profesionalizante, el formato de intervención áulica, el formato de intervención comunitaria (que puede abreviar en modalidades tecnocrático-solidarias o críticas) y un formato que integra las prácticas académicas, encontrando en algunos de ellos la posibilidad de pertenencia a ambos paradigmas, así como cierta ductilidad en algunas herramientas que son reconocidas como mediación de la práctica.

Palabras clave: extensión, prácticas integrales, formato, paradigma, sistematización.

Abstract. This paper analyzes some modalities of the extensionist practice, emphasizing the particularities and the relevance of the economic and socio-political context in which this practice occurs. Categories, such as paradigm, format, device, and tool, are used and discussed in this hierarchical taxonomic system that is defined by two paradigms 
(transferencism paradigm and problematization paradigm), under which the different recognized formats can be grouped: informative format, awareness-raising format, professionalizing format, classroom intervention format, community intervention format (technocratic-solidarity or critical modalities), and a format that integrates the academic practices, finding in some of them the possibility of belonging to both paradigms and the ductility of some tools recognized as mediation of the practice.

Keywords: extension, integral practices, format, paradigm, systematization.

\section{Introducción}

Entre las prácticas que configuran las funciones académicas de las universidades argentinas y latinoamericanas, la extensión universitaria ha comenzado a ocupar, en los últimos años, una centralidad discursiva que es avalada por el lugar que se le otorga en los estatutos, aun cuando pareciera ser la que menos prestigio otorga a las trayectorias académicas, probablemente por la hegemonía y la relevancia que han logrado acumular otras funciones como la investigación o la docencia. A pesar de esto, en los últimos años se ha incrementado el interés en la actividad, traccionando a su vez una fuerte demanda de parte del claustro estudiantil y de algunos docentes y graduados en relación con la importancia de su curricularización como fundamento de la formación de grado y de su jerarquización como práctica (Zavaro, 2019); un proceso que, si bien ocupa un lugar central en los debates actuales, aún constituye una deuda y todo un escenario de disputa.

Por otra parte, la extensión -como el resto de las funciones universitariasestá lejos de constituir una práctica desprovista de neutralidad, sino que es, y está, definitivamente atravesada por un profundo componente político que se insinúa en la letra del manifiesto liminar ${ }^{1}$ como génesis conceptual de una universidad abierta a la comunidad (Tünnermann, 2001), y que, confinada al ostracismo y a ciertas formas de resistencia durante las intervenciones militares en las oscuras épocas de represión y dictadura, ha logrado sobrevivir al clima neoliberal que ha desencadenado diversos procesos de revisión y evaluación de las universidades, adoptando diversos matices y formatos que no solo han contribuido a su diversificación como práctica objetivada, sino también a su reformulación en términos conceptuales.

El Manifiesto Liminar constituye el documento central que explicita los propósitos y las expectativas de la reforma universitaria acontecida en la ciudad de Córdoba en 1918. 
URL: https://www.revistas.una.ac.cr/index.php/dialogo/index

CORREO ELECTRÓNICO: universidadendialogo@una.ac.cr

DOI: https://doi.org/10.15359/udre.10-2.5

En los últimos años, además, en varios países de Latinoamérica y en particular en la Argentina ha habido un notable incremento de propuestas con un fuerte anclaje en las demandas sociales, probablemente como respuesta a una lógica que, atravesada por la necesidad de trabajar en torno al abordaje de problemas concretos, ha comenzado a plantear una nueva lógica fundamentada en una relación de cercanía con el territorio y sus actores.

La extensión entonces, como todas las actividades que configuran la vida académica, no es ajena a las coyunturas socioeconómicas ni a las tradiciones disciplinares, y en esa conjunción se han urdido muy diversos modos en que históricamente se ha ido objetivado, contribuyendo -en ese proceso-a delinear los límites de una identidad que le es propia y que a priori permite distinguirla de otras funciones, aun cuando el debate en relación con la posibilidad y la necesidad de avanzar hacia una práctica integrada comienza a instalarse como uno de los debates centrales.

Bajo esos límites, no obstante, es posible encontrar cierta diversidad de formatos en los que acontece y que dan cuenta de una práctica profundamente polisémica. Establecer entonces una reconstrucción en perspectiva histórica de algunos de estos formatos en una suerte de taxonomía que contribuya a caracterizarlos, definiendo criterios de demarcación, constituye el propósito que vertebra esta reflexión, focalizando en la matriz pedagógica que media en su objetivación y en algunos de los fundamentos epistemológicos que en ellos subyacen.

\section{Los formatos como objetivación de la práctica}

\section{La divulgación del conocimiento como eje de la práctica extensionista...}

Una de las más tempranas experiencias de extensión en la región puede encontrarse en las propuestas de divulgación de las ciencias como eje de la manera de articular con la comunidad. Un tipo de iniciativa que responde a la misión de extender el conocimiento -indescifrable y de intramuros- a la sociedad y que tiene anclaje en una concepción positivista bajo la cual el conocimiento científico es entendido como paradigma del conocimiento, y que supone que quienes no forman parte de la élite académica deben ser iluminados por la razón. La creación de museos dependientes directamente de las facultades, según el campo disciplinar, cuentan entre los primeros aportes, y su agenda, entre las primeras experiencias.

En gran parte de estas instituciones la investigación y el mantenimiento y curatoría de sus colecciones también ha sido fundamental para el desarrollo 
ReVista Universidad en DiÁlogo • Vol. 10, N. 2, Julio-Diciembre, 2020 • 97-124

ISSN 2215-2849 • EISSN: 2215-4752

URL: https://www.revistas.una.ac.cr/index.php/dialogo/index Correo eleCtrónico: universidadendialogo@una.ac.cr

DOI: https://doi.org/10.15359/udre.10-2.5

teórico de las disciplinas, tanto por la contribución que el estudio de estas colecciones ha hecho al conocimiento científico (Crisci y Katinas, 2017) como por el hecho de que atesoran parte del patrimonio cultural de la humanidad, aunque en términos generales el mayor de sus atractivos radica en el diseño de la exhibición, ya sea por la manera en que esta define la lógica del recorrido en relación con aquello que es exhibido o en lo referente a la información que acompaña a cada una de las piezas, y que en los últimos años ha mutado a formas más atractivas y novedosas, desde el punto de vista gráfico, e incluso a modalidades interactivas respecto de la relación que se establece con el visitante y que es resultado de la incorporación de la tecnología a las estrategias de comunicación.

También las publicaciones en revistas orientadas a la divulgación y la popularidad de los documentales que conforman la grilla de muchos canales temáticos o educativos en los que intervienen especialistas de diversas disciplinas de las ciencias naturales (arqueología, paleontología, ecología, física, química o astronomía, etc.), la historia, la ingeniería e incluso la arquitectura, dan cuenta del interés de la población (en particular de la clase media y media alta) por el acceso a este tipo de conocimientos y del creciente interés por parte de la comunidad académica en dar respuesta a esta demanda.

El formato fomenta la resignificación de los saberes que circulan en las aulas universitarias, bajo una perspectiva que es definida como altruista (Serna, 2007) y que denota el intento de los académicos (docentes y estudiantes) por relacionarse -indirectamente o de manera presencial- con la comunidad y que tradicionalmente se ha inscripto, tal como sostienen González y González (2003), en un modo unidireccional de concebir a la comunicación, que termina por reproducir la famosa metáfora de Sherwin Williams como forma particularmente gráfica de representar un modo hegemónico de relación con el conocimiento, al que metodológicamente definimos como transferencista y que se objetiva como una transposición de saberes cuyo eje vertebral lo constituye el lenguaje.

De esta manera, el lenguaje adquiere un valor central al ser entendido como articulador del campo disciplinar que lo delimita y configura y bajo el cual el científico se constituye en aquel sujeto que -siendo conocedor de la temática por su experticia- asume el rol de transmitir y de enseñar a un "otro" al que concibe como destinatario de una información que, para ser comprendida, requiere ser procesada, traducida y "adaptada". Bajo este formato, se concibe a los académicos como portadores de un conocimiento socialmente legitimado con el que es posible "iluminar" a los destinatarios directamente de la fuente del saber. 
URL: https://www.revistas.una.ac.cr/index.php/dialogo/index

CORREO ELECTRÓNICO: universidadendialogo@una.ac.cr

DOI: https://doi.org/10.15359/udre.10-2.5

De la divulgación a la concientización como propósito de la práctica...

Algunos eventos que marcaron la década del setenta en el siglo pasado contribuyeron a delinear un contexto particular en la región y, en consecuencia, a reformular un nuevo modo de relación de las universidades (en su diversidad de disciplinas y saberes) con la comunidad, bosquejando un formato que, además de divulgar el conocimiento, estuvo orientado a generar conciencia en la población. La $1^{\text {ra }}$ Cumbre de la Tierra, celebrada en Estocolmo en 1972, contribuyó a replantear a nivel mundial y regional la problemática del deterioro ambiental como consecuencia del uso irracional de los recursos naturales; también el auge de la filosofía de la liberación en consonancia con corrientes religiosas asociadas al "movimiento en opción por los pobres" contribuyeron a redirigir la mirada de los sociólogos y economistas hacia la problemática de la desigualdad y del analfabetismo en la región, en contraste con la impronta de la Revolución cubana y la sandinista, convertidas -para la izquierda académica- en un horizonte de referencia.

La universidad, como sujeto colectivo, comienza entonces a transitar un camino signado por la posibilidad de despojarse de la perspectiva mesiánica que la había caracterizado hasta ese entonces como herencia del positivismo, y propone despegarse de la extensión literal de los saberes legitimados de intramuros -al menos en relación con lo discursivo-en un intento por provocar las rupturas necesarias con el tradicionalismo transferencista predominante.

Comienza a gestarse un modelo que, según Gezmet (2014), es posible referenciar en las campañas de alfabetización desarrolladas en Brasil durante la gestión de Paulo Freire como ministro de Educación de ese país, y más cercano en el tiempo con algunas campañas relacionadas con la ampliación de derechos o con la salud pública, que forman parte del modo en que en algunas facultades argentinas, por citar un ejemplo, los estudiantes de carreras como Derecho, Medicina, Odontología o Veterinaria se vinculan con la comunidad. Un modelo que sitúa en la centralidad de la escena al sujeto y que, como aporte pedagógico, en oposición a la llamada educación bancaria (Freire, 1970), propone la horizontalidad en los modos de dialogar y construir sentido como estrategia para la emancipación, en una suerte de reivindicación de la dimensión política de la educación.

En este sentido, Serna Alcántara (2007) enmarca a este modelo -al que define como concientizador- en las ideas de la izquierda latinoamericana y la pedagogía freireana, en tanto persigue un despertar de la conciencia, vinculado a un "cambio de mentalidad" que resulta emancipatorio, ya que 
ReVista Universidad en DiÁlogo • Vol. 10, N. 2, Julio-Diciembre, 2020 • 97-124

ISSN 2215-2849 • EISSN: 2215-4752

URL: https://www.revistas.una.ac.cr/index.php/dialogo/index Correo eleCtrónico: universidadendialogo@una.ac.cr

DOI: https://doi.org/10.15359/udre.10-2.5

la "toma de conciencia" podría promover un "despertar" de la sociedad; sin embargo, vincular el valor emancipatorio y transformador del conocimiento con la acción de concientizar a la comunidad sobre un tema en particular resulta al menos contradictorio por cuanto supone el abordaje del problema de un modo unívoco, otorgándoles a quienes ejercen el acto de concientizar el rol de custodiar tales saberes y de asumir una misión que tiene como propósito modificar, ¿colonizar?, la conciencia de un otro que en principio piensa diferente, aun cuando medie el diálogo respetuoso en ese vínculo.

Si bien el propio Freire (1973) al plantear el modo de objetivar la práctica extensionista en ámbitos rurales hace referencia explícita a la concientización, también destaca los peligros existentes en esa acción, discerniendo las diferencias entre la conciencia intelectualizada y aquella conciencia que es objetivada por la experiencia, y aclarando la posibilidad de que en ese esfuerzo se termine imponiendo un saber por sobre otro que es prescripto y manipulado en esa interacción, en cuyo caso la posibilidad de emancipación no es sino una farsa.

Concientizar sin sentirse interpelado por la singularidad del otro y de su realidad remite -indefectiblemente- a una perspectiva transferencista, con la particularidad de que esta modificación de conciencias es legitimada por la superioridad que encierra el corpus de conocimientos que se intenta instalar en la estructura de conceptos de aquellos sujetos a quienes se pretende concientizar. Si bien los motivos que subyacen usualmente en el diseño de una campaña o de una estrategia de concientización en muchas oportunidades es más que loable e incluso podría incidir de manera directa en un beneficio colectivo, el acto mismo de arrogarse la imposición de una idea -por más acertada que la consideremos- constituye un acto de colonización simbólica de la racionalidad de aquellos a quienes consideramos deben reproducir nuestros modos de ver e interpretar la realidad.

En las antípodas, el encuentro de saberes representa un vínculo más sincero con la comunidad, que permite articular historias de vida, trayectorias formativas y modos de entender el mundo y que apuesta a la concreción de acuerdos entre sujetos en paridad de condiciones, lo que contribuye en cierta medida a desdibujar las fronteras disciplinares que "encorsetan" y disciplinan la creatividad, promoviendo un nuevo conocimiento que no es resultado de la imposición, sino de la comunicación recíproca y de la reflexión colectiva. Esta visión de la extensión ha contribuido a transformar paulatinamente la manera de entender desde la academia el contacto con el "otro", facilitando un modo más dialógico y natural de inserción de las universidades en el territorio. 
URL: https://www.revistas.una.ac.cr/index.php/dialogo/index

CORREO ELECTRÓNICO: universidadendialogo@una.ac.cr

DOI: https://doi.org/10.15359/udre.10-2.5

\section{La extensión rural como práctica profesionalizante...}

En sintonía con este formato concientizador, es interesante destacar la impronta que en el ámbito rural ha tenido la actividad extensionista, con un vínculo que se remonta, rastreando la historia de los sistemas agrícolas -al menos en Argentina (Sánchez, 2012)-, a la llamada revolución verde, en el marco de las teorías del desarrollo (Regidor, 1987), bajo el supuesto de que la transferencia del paquete tecnológico resultante de la innovación de los países centrales en relación con maquinarias, semillas o agroquímicos favorecería la modernización del sistema productivo en los países subdesarrollados o en aquellos en vías de desarrollo, garantizando el aumento de la productividad y las ganancias en [una fracción de] el sector.

Incorporar este tipo de tecnologías a la producción ha requerido, en principio, de mediadores capaces de explicitar las bondades del paquete. Esto es, profesionales formados que sean capaces de asesorar a los productores en la adopción y transición a este modelo productivo. Un modo de dimensionar las concepciones históricas asociadas a esta perspectiva radica en la paráfrasis que Ceccon (2008, p. 24) hace al recrear el pensamiento de Theodore Schultz, uno de los ideólogos de la revolución verde, quien, resumiendo la impronta del movimiento, define al agrónomo como "una persona que iba a civilizar al sujeto de pies descalzos, al bárbaro que se encontraba en íntimo contacto con la naturaleza, pero sometido a ella [cursivas añadidas]".

Esta perspectiva transferencista que subyace en el planteo, impactaron en la modificación de los planes de estudio en diferentes universidades, incorporando materias y saberes que hasta el momento no formaban parte de las tradiciones disciplinares de un agrónomo, de un veterinario o de un ingeniero forestal, e incluyó saberes que pusieron en el centro del proceso de transformación y, por ende, en el foco de la currícula a los productores como destinatarios directos a los que es preciso acompañar en una transición que -definitivamente-no es neutral. Esta coyuntura incluso abrevó en varias facultades en la creación de materias enfocadas en la extensión rural como práctica disciplinar, una práctica que, si bien no resume los fundamentos de la extensión universitaria como función académica, sí ha sido entendida en el imaginario de docentes y estudiantes de ciencias agrícolas y afines como tal, o al menos como un modo de formar a los futuros egresados en el trabajo territorial y en el vínculo con la sociedad.

Claramente, el tema amerita un análisis más profundo que excede el propósito de estas líneas, pero aun en medio del cambio conceptual que comienza a instalarse en la sociedad en favor de la soberanía alimentaria y de nuevos 
ReVista Universidad en DiÁlogo • Vol. 10, N. 2, Julio-Diciembre, 2020 • 97-124

ISSN 2215-2849 • EISSN: 2215-4752

URL: https:/www.revistas.una.ac.cr/index.php/dialogo/index Correo electrónico: universidadendialogo@una.ac.cr

DOI: https://doi.org/10.15359/udre.10-2.5

modos de producción que distan de los orígenes de la revolución verde y de la transferencia tecnológica, la extensión rural ha formado parte de los trayectos formativos de los profesionales del agro desde hace varias décadas y lo continúa siendo en la actualidad. No obstante, y a pesar de que esta formación ha estado permeada a partir de la década del setenta por la influencia de las ideas de Freire (1973) en relación con la relevancia de la perspectiva dialógica en torno al vínculo entre los diversos actores (productor, profesional, extensionista), el modo en que acontece ha estado enfocado -en muchas de las experiencias acreditables- en la concientización del trabajador rural y en la asistencia técnica de productores e inversores, más que en la reflexión en torno a los modos de producción y a su impacto en las condiciones de vida del peón rural, de los pequeños y medianos productores y de la economía en general, temas que comienzan a aparecer en iniciativas más recientes, inaugurando un camino de transformación que tiene origen en un paradigma transferencista y tecnocrático y horizonte en un nuevo modo de problematizar este vínculo que, claramente, excede a la extensión universitaria como práctica académica.

\section{La construcción colectiva y el aula como espacio de articulación}

En los últimos años ha proliferado como propuesta de extensión -al menos en Argentina- un tipo de vínculo entre las universidades y las escuelas, que integran el sistema educativo preuniversitario (enseñanza primaria y secundaria), que constituye un modo de "intervenir" el aula de pregrado. Una de las particularidades de este formato, que logra sacar a los docentes y estudiantes de las aulas universitarias para irrumpir en aquellas en que se educan los chicos de niveles educativos previos, ha sido la articulación curricular como eje de la propuesta de vinculación entre ambas instituciones, aunque bajo la premisa generalizada en una parte de la comunidad académica, y en gran parte de la sociedad, de que la escuela (en especial la pública) está atravesando una crisis institucional que constituye la causa de que los ingresantes lleguen a la universidad sin una preparación adecuada para el nivel superior que comienzan a cursar, ni en contenidos, ni en herramientas metodológicas o habilidades (como la comprensión de textos o la escritura, etc.), ni en valores; aunque sí han logrado adaptarse a la utilización de atajos como estrategia de evasión de conocimientos, un fenómeno que, Ortega (2011) aclara, no es patrimonio de un sector social en particular, sino que más bien abreva en una cultura del pragmatismo y de la indiferencia a la que Bauman (2004) adjetiva como modernidad líquida. 
URL: https://www.revistas.una.ac.cr/index.php/dialogo/index

CORREO ELECTRÓNICO: universidadendialogo@una.ac.cr

DOI: https://doi.org/10.15359/udre.10-2.5

Uno de los prejuicios que históricamente han primado respecto del sistema educativo formal responde al supuesto de que la educación preuniversitaria acontece bajo un modelo de tipo conductista. Si bien el conductismo se remonta a las ideas de Watson (1913) dentro del campo de la psicología y se fundamenta en un tipo de relación causa-efecto, que presupone el condicionamiento de la respuesta ante un determinado estímulo cognitivo, en el campo pedagógico estas ideas abrevan en las expectativas que atraviesan la práctica docente y que suponen que ante la enseñanza de un contenido es de esperar una determinada respuesta por parte del estudiante en relación con aquello que es enseñado y, en consecuencia, aprendido y susceptible de ser evaluado.

El modelo comunicacional instalado en esta relación docente-alumno es claramente lineal y unidireccional, porque el docente constituye el único emisor de un mensaje que resigna a los estudiantes a ocupar un rol de meros receptores de la información, fomentando un aprendizaje de tipo memorístico en el que no parece ser relevante la resignificación de conocimientos, sino su reproducción, y bajo el cual la evaluación usualmente se convierte en un obstáculo para el aprendizaje, ya que la normativa institucional establece de manera rígida plazos en los que los alumnos deben demostrar, a través de un examen, que los contenidos del programa han sido aprendidos, como si el aprendizaje pudiera limitarse a un determinado periodo de tiempo (Moreno, 2011), olvidando que tanto la enseñanza como el aprendizaje constituyen procesos cognitivos diferentes (Kaplún, 2005). Esto promueve que los estudiantes estudien para "aprobar" los exámenes y acreditar la materia, y no para aprender, desvalorizando en este proceso el valor mismo del conocimiento.

La intervención a través de las prácticas extensionistas en las aulas de los colegios muchas veces también reproduce el modelo transferencista, porque tampoco las aulas universitarias, lamentablemente, están lejos de ese modo de enseñanza, aunque en muchos equipos con amplia trayectoria en la temática ha ido fecundando una perspectiva constructivista en el modo de tejer saberes entre aquellos sujetos que participan de este tipo de experiencias, en las que el conocimiento previo es revalorizado como forma de encuentro en relación con el abordaje de los temas y en los cuales la circulación de la palabra se constituye en el centro de la comunicación y de la construcción colectiva de conocimientos.

A pesar del carácter no formal de estas dinámicas, su diseño en términos metodológicos no deja lugar a la improvisación, por el contrario, requiere una exhaustiva planificación donde no solo hay lugar para la diversidad de 
ReVista Universidad en DiÁlogo • Vol. 10, N. 2, Julio-Diciembre, 2020 • 97-124

ISSN 2215-2849 • EISSN: 2215-4752

URL: https://www.revistas.una.ac.cr/index.php/dialogo/index Correo eleCtrónico: universidadendialogo@una.ac.cr

DOI: https://doi.org/10.15359/udre.10-2.5

opiniones, sino también para la implementación de herramientas lúdicas que operan como estrategia de vinculación entre los sujetos y como un instrumento que favorece la mediación de la palabra. Si bien para algunas de las tradiciones del sistema formal esto podría suponer una suerte de disrupción o de dispersión de la enseñanza y, en consecuencia, de la planificación original de las clases, el uso de estas metodologías queda comprendido bajo el universo de las macrodecisiones implícitas en la construcción metodológica (Edelstein, 1996), que configura el marco teórico-pedagógico bajo el que es posible inscribirlas.

Entender al aula entonces no solo como un espacio físico destinado al aprendizaje de contenidos conceptuales que definen cada una de las materias que integran la currícula escolar, sino también -y sobretodo- como un espacio de interacción social en el cual se explicitan formas de pensar, sentir, expresarse y actuar (Jeria, 2013) como parte de un aprendizaje colectivo es una de las miradas que justifica y revaloriza el trabajo desde las universidades con las escuelas, especialmente cuando es posible lograr una articulación de tipo simbiótica que es capaz de garantizar la construcción de vínculos sostenibles en el tiempo, que en su mayoría representan para las escuelas y para sus docentes un puente que fomenta el intercambio de conocimientos disciplinares, la capacitación y la actualización, constituyendo - en paralelo- una forma de apoyar y de acompañar desde las universidades el desarrollo y la instrumentación de la currícula escolar, como contribución al proceso de transformación de la enseñanza preuniversitaria.

Para los extensionistas universitarios, por su parte, intervenir en la currícula escolar constituye una manera activa, concreta y creativa de resignificar contenidos propios del plan de estudio de sus carreras y de encontrar un espacio de aprendizaje contextualizado que les represente un reto real, al estar en contacto con la comunidad y con las problemáticas que las atraviesa y que usualmente exceden la formación profesional, lo que inevitablemente ha de impactar en su formación como personas y futuros profesionales. Como saldo, la posibilidad, para quienes participan, de transitar por nuevas formas de un aprendizaje interactivo y socializado que potencia la transformación de los contenidos curriculares en saberes resignificados por la experiencia e incorporando estrategias no formales, que desde el punto de vista didáctico constituyen un aporte interesante al sistema educativo en niveles de enseñanza como el secundario, donde, por las características propias de la edad de los estudiantes, el juego es considerado como algo distante y bochornoso.

Además, este acercamiento de las universidades a las escuelas, especialmente en el nivel secundario, permite incidir favorablemente en la formación voca- 
URL: https://www.revistas.una.ac.cr/index.php/dialogo/index

CORREO ELECTRÓNICO: universidadendialogo@una.ac.cr

DOI: https://doi.org/10.15359/udre.10-2.5

cional y esto contribuye a facilitar el acceso de los chicos a las aulas universitarias como posibilidad de continuar estudios superiores, fomentando de esta manera la movilidad social -en especial cuando se trata de escuelas de barrios periféricos donde los estudios universitarios usualmente no son considerados por los jóvenes entre sus expectativas de futuro inmediato-, lo que impacta de manera positiva en la acumulación de capital cultural (Bourdieu, 1987) tanto a nivel personal como social.

\section{La extensión en la organización comunitaria y la transformación social...}

Algunas de las experiencias heredadas de la militancia barrial han ido configurando a lo largo de estos años, al menos en Argentina, un modo de intervención territorial, colaborativo y solidario donde la presencia de la universidad en los barrios más postergados permitió desarrollar formas de articulación encaminadas a la búsqueda de soluciones en las que el conocimiento disciplinar toma relevancia en la acción. En esta línea de trabajo ha sido central el rol de los referentes, conocedores del contexto, como facilitadores de un proceso que apuesta a generar empatía con los vecinos como estrategia para el abordaje de las problemáticas.

Bajo este formato han coexistido diversas maneras de articulación que a menudo han estado atravesadas por las concepciones e ideologías de quienes conforman los equipos de trabajo y que de alguna manera han definido los modos de relación entre los actores. En muchos casos la presencia de la universidad en el territorio representa para los vecinos una referencia muy fuerte en términos de legitimación de saberes y esto constituye todo un reto en lo que respecta a los modos de vinculación, por la tentación de caer en el facilismo de imponer un formato transferencista donde las soluciones responden a una lógica puramente tecnocrática.

Algunas de las temáticas más frecuentes han incluido, e incluyen, las huertas alimentarias (ya sean familiares o comunitarias) como modo de proveerse de alimentos en condiciones donde la seguridad alimentaria es una utopía, o la existencia de basurales a cielo abierto que muchas veces están relacionados con el desecho de la basura en lugares donde no suele haber una recolección frecuente de los residuos por parte del Estado o que es resultado del cirujeo que algunos vecinos desarrollan como trabajo y que usualmente apunta al reciclado, la clasificación y la separación de estos para la venta. 
ReVista Universidad en DiÁlogo • Vol. 10, N. 2, Julio-Diciembre, 2020 • 97-124

ISSN 2215-2849 • EISSN: 2215-4752

URL: https://www.revistas.una.ac.cr/index.php/dialogo/index Correo electrónico: universidadendialogo@una.ac.cr

DOI: https://doi.org/10.15359/udre.10-2.5

Otros temas cotidianos de intervención han estado relacionados con problemáticas sanitarias en relación con el control de la natalidad y el uso de anticonceptivos, la vacunación, el control de vectores de enfermedades y parasitosis o el abordaje de problemáticas relacionadas con algunos factores de deterioro ambiental respecto de la flora y fauna local, la contaminación, el mascotismo o a la castración de perros y gatos como una manera de contribuir en cierta forma al mejoramiento de la calidad de vida individual y ciudadana de la comunidad, incluso en paralelo a "campañas de concientización" como las referidas en párrafos anteriores.

En contraposición a este modo de vinculación tecnocrático, aparece una modalidad que puede denominarse como problematizadora, que, si bien apuesta a la resolución de una misma problemática, propone como proceso la elaboración colectiva de diagnósticos participativos y dialógicos a partir de los cuales sea posible consensuar diversas estrategias de solución. Si bien no siempre esta solución depende de los actores involucrados, el análisis crítico y la participación colectiva fomentan el intercambio de experiencias, ideas y saberes, como parte de un proceso en el que el debate se convierte en una estrategia que permite modular disensos y consensos, amalgama intenciones y voluntades y en el cual el taller -como dispositivo de trabajo- se convierte en un espacio que opera como ordenador de la construcción colectiva de conocimientos, de la planificación y de la toma de decisiones.

Bajo este formato la comunidad no es entendida como "destinataria" de la práctica extensionista, sino que se convierte en miembro activo de un proyecto que se elabora y planifica en respuesta a las demandas que emergen del diagnóstico. En este contexto los extensionistas tampoco son transmisores de saberes instituidos, sino facilitadores del vínculo, y para ello es fundamental su formación en relación con la adquisición de herramientas que faciliten y orienten el trabajo territorial.

De esa manera, la perspectiva colaborativa y solidaria ha ido transmutándose en el tiempo en un modo profundamente racional de conectarse con el otro, con los problemas que lo atraviesan y con la coyuntura que los condiciona, aunque lejos de centrar el objetivo en la solución técnica de las problemáticas, se enfoca en intentar lograr una aproximación crítica a la realidad, que incorpore una lectura de la complejidad que la caracteriza, categoría que ha sido ampliamente discutida por Leff (2007), y bajo la cual se incorpora a la discusión de las problemáticas concretas no solo las causas que las condicionan, sino también las posibles consecuencias de las acciones a desarrollar, la responsabilidad que le concierne a cada uno de los actores involucrados y las diferentes estrategias 
URL: https://www.revistas.una.ac.cr/index.php/dialogo/index

CORREO ELECTRÓNICO: universidadendialogo@una.ac.cr

DOI: https://doi.org/10.15359/udre.10-2.5

de abordaje, entendiendo al ambiente como una construcción social con toda la complejidad que esto supone.

Además, esta posibilidad de consensuar ejes de trabajo no solo permite tomar decisiones en conjunto que contribuyan a encauzar las soluciones a los problemas, sino que apuestan a fomentar el compromiso personal con el (y los) otro(s) y con la organización comunitaria, a partir de un debate sostenido, donde la democratización de la palabra constituye uno de los fundamentos pedagógicos centrales de una práctica en la que los estudiantes "universitarios" trabajan conjuntamente con los vecinos, aprendiendo a investigar la realidad con ellos, utilizando métodos propios de la investigación-acción participativa, lo que inevitablemente contribuye, en ambos sentidos, a la formación de sujetos sentipensantes (Fals, 2009) y críticos como camino hacia el empoderamiento y la emancipación.

\section{Configuraciones en la sistematización de la práctica...}

\section{La taxonomía como organizador...}

Tal como analizamos con anterioridad, han sido muy diversos los modos en que se ha ido constituyendo históricamente la extensión universitaria como práctica académica y ha sido la propia práctica, probablemente, la que ha permitido ir configurando los límites del campo que, reconociendo su historicidad y la relevancia de los contextos en que se objetiva, comienza a incorporar nuevos formatos, a definir sustratos y sustentos pedagógicos que orientan los modos de relación con el conocimiento y con la comunidad y, a su vez, a excluir en ese proceso otras prácticas que definitivamente no califican dentro de los parámetros de lo que hoy comienza a ser reconocido y delimitado como extensión, aun cuando esta constituye, no obstante, una práctica profundamente polisémica.

En este sentido, y en un intento por establecer una suerte de taxonomía acerca del modo en que se objetivan las prácticas en atención a sus fundamentos pedagógicos y a los propósitos que las sostienen, apelaré a algunos metaconceptos como ordenadores de esta diversidad: paradigma, formato, modalidad, dispositivo y herramienta son utilizados como categorías que permiten aproximarnos a su comprensión.

Bajo esta sistematización, la categoría de paradigma -entendida como la más inclusiva- es definida como aquel sistema de supuestos y de fundamentos teóricos, epistemológicos y pedagógicos que orientan y contienen una práctica 
Revista Universidad en DiÁlogo • Vol. 10, N. ํ 2, Julio-Diciembre, 2020 • 97-124

ISSN 2215-2849 • EISSN: 2215-4752

URL: https://www.revistas.una.ac.cr/index.php/dialogo/index CORREO ELECTRÓNICO: universidadendialogo@una.ac.cr

DOI: https://doi.org/10.15359/udre.10-2.5

que es capaz de aglutinar y expresarse en diversos formatos y modalidades, demarcándolos. Bajo un mismo paradigma entonces es posible reconocer diversos formatos delimitados por el modo en que se explicitan y que fueron analizados en la reconstrucción previa, como parte de una trazabilidad que da cuenta de la propia historia del campo y que, a su vez, configura dispositivos que constituyen el núcleo de la objetivación de las actividades y acciones que son diseñadas y planificadas en el marco de configuraciones con propósitos y objetivos particulares, y en las que es posible utilizar y engarzar muy diversas herramientas ${ }^{2}$ que operan como mediadoras de la acción.

Como rasgo relevante cabe señalar que estas herramientas no necesariamente son exclusivas de un formato en particular, lo que contribuye a configurar una suerte de ecología de modalidades que relativizan incluso los límites entre los formatos, desdibujándolos en algunos casos, lo que en cierto modo "salva" a esta suerte de arreglo jerárquico del reduccionismo del que podría adolecer cualquier sistema de clasificación, complejizando y flexibilizándolo.

En este intento por sistematizar la diversidad de experiencias en extensión, es posible reconocer entonces paradigmas que hoy conviven en la academia y que coinciden con lo que Tommasino y Cano (2016) llaman modelos en extensión. A modo de aclaración y con la intención de justificar el sistema de jerarquías que les atribuyo a las categorías propuestas y que reasigna a estos modelos la categoría de paradigma, creo necesario retomar una definición de Gezmet (2014) que define al modelo como aquel recurso metodológico que permite conocer, interpretar o explicar los componentes más relevantes que organizan y estructuran conjuntos de nociones, ideas, ideologías y prácticas. El término paradigma, en cambio, engloba todo un sistema de creencias y supuestos (Flores, 2004) que se traducen en el modo de concebir la relación del sujeto cognoscente con todo aquello que lo rodea y que forma parte de la realidad en la que se encuentra inmerso, lo que no solo permite interpretar el mundo, sino también formular aquellas preguntas que configuran el marco teórico de referencia (Kuhn, 1962), desde donde es posible producir lecturas de la realidad que son legitimadas en la práctica, tanto por el modo en que metodológicamente se concreta esa aproximación como por aquellas

\footnotetext{
Al utilizar el término herramienta en el contexto de la presente taxonomía sobre las prácticas de extensión nos referimos a diferentes recursos o mediadores de la práctica como charlas, dispositivos gráficos, juegos, Power Point, dinámicas de grupo, etc. Las herramientas no son privativas de un único formato ni de un paradigma, sino que es este último el que determina el modo en que se utilizan e insertan en el dispositivo, según el propósito que se persigue y la lógica de la interacción que caracteriza a un formato en particular. Debe ser excluida del concepto de herramienta -aun cuando en algunos casos parezca sutil y ambiguo- la referencia a materiales didácticos.
} 
URL: https://www.revistas.una.ac.cr/index.php/dialogo/index

CORREO ELECTRÓNICO: universidadendialogo@una.ac.cr

DOI: https://doi.org/10.15359/udre.10-2.5

conclusiones e intersubjetividades que son aceptadas bajo el conjunto de supuestos que condicionan la lectura del contexto y de la propia práctica.

Más allá de la discusión en torno a la pertinencia y jerarquía sistémica de la categoría más adecuada para enmarcar el conjunto de supuestos que configuran estas dos maneras de aproximarse a la lectura de la realidad y a establecer vínculos con ella -y por lo tanto con la sociedad-, el [modelo] paradigma transferencista reproduce tradiciones disciplinares bajo las cuales la extensión es entendida como una prolongación del conocimiento académico que persigue "iluminar", concientizar e incluso trabajar de modo colaborativo con la sociedad, pero a partir de una perspectiva tecnocrática donde el conocimiento legitimado es el académico.

Los formatos contenidos bajo esta perspectiva de la extensión deben distinguirse, tal como señala Langer (2010), tanto de la transferencia de conocimientos que se espera del desempeño de un graduado universitario en actividades de asesoramiento o de consultoría a empresas durante el ejercicio de su profesión como de la transferencia tecnológica bajo el llamado modelo de triple hélice (González de la Fe, 2009), que conjuga la relación existente entre las universidades, el Estado y la industria en lo referente a la aplicación tecnológica, a pesar de que en todos los casos el denominador común lo constituya el contexto de aplicación del conocimiento producido en la academia, pero resignificado en nuevos y diferentes ámbitos. Bajo el paradigma transferencista, entonces, la extensión se constituye en contexto de aplicación de un conocimiento académico que, tal como describe Chevallard (1998) respecto a la transposición didáctica, constituye un saber exiliado de sus orígenes y de su producción histórica en la esfera del saber sabio e implantado en un nuevo escenario como saber enseñado.

En contraposición, el otro de los paradigmas, al que denominamos problematizador, y que Tommasino y Cano (2016) definen como modelo de extensión crítica, se sustenta en una problematización de la realidad como fundamento de su práctica y se objetiva con base en posiciones constructivistas que revalorizan la democratización de la palabra y la confluencia dialógica de saberes de diferente extracción y naturaleza, eincluye tanto trayectorias disciplinares como experiencias de vida. Ambas se amalgaman en un proceso colectivo que es legitimado por la intersubjetividad que emerge del diálogo y del diagnóstico participativo de la realidad. Bajo este paradigma uno de los ejes centrales que encauzan el propósito de la práctica es el proceso de empoderamiento de la comunidad y el valor emancipatorio que se le asigna al conocimiento. 
ReVista Universidad en DiÁlogo • Vol. 10, N. 2, Julio-Diciembre, 2020 • 97-124

ISSN 2215-2849 • EISSN: 2215-4752

URL: https://www.revistas.una.ac.cr/index.php/dialogo/index Correo eleCtrónico: universidadendialogo@una.ac.cr

DOI: https://doi.org/10.15359/udre.10-2.5

Tal como hemos analizado con anterioridad, existe una gran diversidad de modalidades en que se concretan y visibilizan las prácticas de extensión, que pueden ser reconocidas -en el presente sistema- bajo la categoría de formato, en atención a aquellas regularidades que los delimitan y definen, aun cuando es posible encontrar zonas transicionales cuyos matices dificultan una demarcación inequívoca, probablemente porque la extensión se constituye en una práctica dialéctica que es desarrollada por sujetos que inevitablemente aportan sus modos personalísimos de interpretar la realidad, configurando en su objetivación una urdimbre de subjetividades que están moduladas por la confluencia de experiencias, tradiciones, convicciones e historias personales, en un contexto que es permanentemente cambiante.

No obstante, esta diversidad abreva en uno u otro de los paradigmas enunciados, y si bien la utilización de algunas herramientas es más frecuente bajo un paradigma que otro, los formatos no son estrictos ni excluyentes en su metodología. Si se conformase un gradiente definido por dos ejes en el espacio, que se corten entre sí conformando una suerte de mapa de cuadrantes donde pueda ubicarse a cada uno de ellos, y en el que uno de los ejes se corresponda con los supuestos epistemológicos que sustentan a cada uno de los paradigmas (problematizador vs. transferencista) y el otro con aquellos propósitos (concientización de la sociedad vs. emancipación) que en términos pedagógicos los definen, podemos ahondar en algunas de estas diferencias y convergencias que los caracterizan.

En principio, es posible reconocer al menos seis formatos, de los cuales cinco han sido esbozados en párrafos anteriores. Aquellas propuestas basadas en la divulgación del saber sabio (formato divulgativo) inevitablemente se inscriben bajo el paradigma transferencista con el claro propósito de acercar el conocimiento científico a la población y/o concientizar a la comunidad en aquellos temas que son considerados -en opinión de los extensionistas- como necesarios para el mejoramiento de la calidad de vida (formato concientizador). El formato de la intervención áulica, que incluye propuestas no formales en instituciones educativas preuniversitarias, resulta ambivalente en lo que respecta a los paradigmas desde los que se plantea el trabajo con alumnos y docentes, y si bien cada vez es más frecuente la utilización de herramientas lúdicas que son propias de corrientes como el constructivismo, más que de un modelo educativo tradicional y conductista, los modos de intervención son diversos y a menudo no suelen apostar como objetivo central -incluso por la discontinuidad de las intervenciones-a revalorizar el valor emancipatorio del conocimiento, sino al conocimiento en sí mismo. 
URL: https://www.revistas.una.ac.cr/index.php/dialogo/index

CORREO ELECTRÓNICO: universidadendialogo@una.ac.cr

DOI: https://doi.org/10.15359/udre.10-2.5

Por otra parte, los modos colaborativos de trabajo (formato tecnocrático solidario) suelen tener una impronta tecnocrática, más pragmática y transferencista que difiere de las prácticas territoriales problematizadoras (formato sociocomunitario crítico), porque el objeto central de esta última apela, más que a la búsqueda de soluciones inmediatas, a la emancipación personal y colectiva desde la participación reflexiva, desarrollando capacidades que permitan transformar la realidad cotidiana y, en ese proceso, lograr la transformación de la comunidad en un colectivo crítico, autónomo y organizado.

Más allá de las diferencias teóricas existentes entre estos formatos, los matices desdibujan las fronteras que puedan existir en sentido estricto, lo que en muchos casos es resultante de la pluralidad de aportes y miradas de los integrantes que conforman los equipos de trabajo y de sus interacciones con la totalidad de participantes que involucra la práctica, ya que cada uno de los sujetos trasunta inevitablemente, durante este proceso, sus modos de concebir y de habitar la universidad (Bourdieu, 2008), y ese habitus contribuye a reproducir y configurar las tradiciones disciplinares, que incluso son paulatinamente reformuladas por el impacto de la heterogeneidad que caracteriza a los equipos y por la hibridación de conocimientos (Vaccarezza, 2015) que se produce como parte de un encuentro entre trayectos académicos disímiles y entre estos y las trayectorias de los miembros de la comunidad con quienes se vinculan.

Además, la inteligibilidad, que según Fresán Orozco (2004) se le atribuye a la extensión tanto por parte de la sociedad como por la comunidad académica, ha contribuido a reconocer en esta, tal como adelantamos, una práctica polisémica, aun cuando en los últimos años algunos formatos han comenzado a imponerse por sobre otros que comienzan a ser cuestionados y que, si bien siguen siendo legitimados por la academia, en los últimos años han comenzado a ser desplazados a las fronteras del propio campo, ocupando la centralidad de un debate que comienza a considerarlos como una función diferente con propósitos y límites propios (Langer, 2010), lo cual le otorga una nueva identidad a ciertas prácticas - como la extensión rural ${ }^{3}$ - que históricamente fueron entendidas bajo el campo de la extensión.

La diversidad de sentidos en torno al significante del término en sí mismo y a la diversidad de actividades que aún hoy son legitimadas desde la práctica,

La extensión rural, siendo una práctica asociada a ciertas particularidades de la profesión de las ciencias agrarias y veterinarias, es excluida del presente arreglo taxonómico y considerada, tal como se percibe en algunos debates disciplinares, como una actividad de transferencia de conocimientos que excede la extensión como función académica. 
incluso antagónicas en su concepción, convierten además a la extensión en un escenario de disputa que supera lo pedagógico y que alcanza el territorio de la política universitaria. En este sentido, coincido con la categoría enunciada por Laclau y Mouffe (2011) en relación con el significante vacío que es desarrollado por Tommasino y Cano (2016).

\section{El apego a las tradiciones...}

Además del rasgo polisémico de la extensión, otro de los ejes que contribuye a desdibujar las fronteras de los formatos anteriormente discutidos son las tradiciones y contribuciones metodológicas propias de cada una de las disciplinas que son arrastradas a la centralidad de la práctica por quienes desarrollan este tipo de actividades, sin lograr despojarse del habitus que supone su formación en una rama determinada del conocimiento. Las didácticas disciplinares, que son naturalizadas como parte de las tradiciones propias de las tribus académicas (Becher, 1993), contribuyen a definir diversos modos de comprensión de la realidad a partir de la subjetividad de quienes conforman el propio campo y que inevitablemente impactan en los modos de relacionarse, tanto con aquellos que lo constituyen como con quienes quedan excluidos por diversas razones de los límites del mismo.

En este sentido, es conveniente establecer como criterio de demarcación en relación con el positivismo - que asume la existencia de una realidad objetivala relevancia que ocupa la subjetividad en la lectura de la realidad y la relación existente entre la producción de conocimiento científico, su provisionalidad y la didáctica propia de cada disciplina, que a su vez define y condiciona los modos en que circulan estos saberes entre los sujetos involucrados en los procesos de socialización, aun bajo paradigmas diferentes, como puede ser el transferencista o el paradigma crítico o problematizador.

\section{El fin de la metáfora...}

Los objetivos vertebrales a los que nos referíamos en la introducción han alentado -al menos en las universidades argentinas- una metáfora donde la docencia (que ha constituido la función fundamental tanto de las universidades privadas como de las estatales), la investigación y la extensión constituyen "patas" de una misma mesa (Zavaro, 2019), a pesar de que no todas son consideradas universidades de investigación, ni la extensión es una prioridad para algunas de ellas e incluso porque en otras instituciones la transferencia tecnológica comienza a configurar nuevas tradiciones, conformando una mesa 
URL: https://www.revistas.una.ac.cr/index.php/dialogo/index

CORREO ELECTRÓNICO: universidadendialogo@una.ac.cr

DOI: https://doi.org/10.15359/udre.10-2.5

de cuatro patas (o quizás más, si se tiene en cuenta la relevancia que en la universidad revisten otras funciones como la gestión).

La relación entre sujeto y objeto de conocimiento ha configurado a lo largo del tiempo los diversos modos en que se objetivan algunas de estas prácticas, en especial la investigación científica o la extensión universitaria. La tradición positivista ha logrado establecer límites precisos entre estas, que han sido definidos en relación con el rol que ocupa el objeto de estudio respecto de la pregunta que elabora un sujeto (investigador) para abordar, de la manera más objetiva y bajo un marco teórico definido por el programa de investigación, la resolución de un problema. No obstante, la investigación no es estrictamente objetiva por estar atravesada por la influencia inevitable de quienes formulan las hipótesis de trabajo y diseñan la metodología "apropiada" para contrastarlas, porque las hipótesis no son neutrales, y más aún porque muchas veces la distancia entre el sujeto que investiga y el objeto de estudio no es tan simple de circunscribir ni de acotar, por tratarse -en algunas disciplinas- de otros sujetos con quienes inevitablemente se establecen vínculos que contribuyen a desdibujar los límites entre campos.

Para disciplinas como la astronomía o la física cuántica, por citar ejemplos, el objeto de investigación bajo el contexto de justificación en que se produce conocimiento científico suele estar sumamente distante de las problemáticas cotidianas que atraviesan la vida en un barrio, por lo que este conocimiento bajo un contexto de aplicación suele limitarse a la transferencia tecnológica (en caso de que sea posible), o bien, en relación con la extensión, a una suerte de transposición didáctica que abreva en formatos de tipo divulgativo o de intervención educativa.

En contraposición, desde otras disciplinas como el trabajo social, la etnografía o la sociología los límites entre estas prácticas académicas suelen diluirse, por cuanto el objeto de investigación lo constituyen otros sujetos que indirectamente inciden en el modo en que se estructura la investigación, a tal punto que esta termina fomentando otros modos de relación que exceden a la pregunta inicial y que incluso fomentan la transmutación de una hipótesis explicativa (Klimovsky, 1971) bajo un escenario de contrastación, en lo que Barraza Macías (2010) denomina hipótesis de acción y que constituye uno de los supuestos claves del paradigma problematizador.

De esta manera, bajo un paradigma transferencista, la extensión suele ser concebida como contexto aplicación [transposición] didáctica de los saberes disciplinares, en tanto que bajo formatos críticos o problematizadores estos 
ReVista Universidad en DiÁlogo • Vol. 10, N. 2, Julio-Diciembre, 2020 • 97-124

ISSN 2215-2849 • EISSN: 2215-4752

URL: https://www.revistas.una.ac.cr/index.php/dialogo/index Correo eleCtrónico: universidadendialogo@una.ac.cr

DOI: https://doi.org/10.15359/udre.10-2.5

saberes suelen ser un punto de partida para la construcción colectiva de un tipo de conocimiento que vincula tanto la dinámica propia de las disciplinas como las experiencias de vida en la solución de problemáticas territoriales.

Esta articulación de saberes y propósitos supone un nuevo formato reconocido bajo la denominación de las prácticas integrales, que en ciertamanera recapitulan la concepción de Sousa Santos (2007) sobre el conocimiento pluriuniversitario. Bajo esta mirada se diluyen varios de los formatos discutidos con anterioridad y confluyen algunas de las prácticas que, como eje común, revalorizan el conocimiento como construcción de la realidad, se resignifica el rol de los sujetos en la producción de conocimientos y se contextualiza el objeto de estudio, de tal modo que la disociación aparente en la diversidad de tradiciones universitarias termina amalgamándose en un formato que integra modos de producir conocimiento y de socializarlo, y que propulsan por deconstruir la famosa metáfora sobre "las tres patas de la mesa", que ha sido imbricada en la subjetividad de la comunidad universitaria con base en sus objetivos explícitos, aun cuando Baptista y Kuenzer (1999) han sentado precedente en la discusión respecto del principio de la indisociabilidad de estas funciones como forma de garantizar la transformación crítica de las universidades.

Profundizando en esta lógica, Kaplún (2005) plantea la necesidad de indisciplinar la universidad y de este modo contribuir a desdibujar los límites que han encorsetado la lectura racional del mundo en torno a un problema de investigación acotado por las tradiciones, los marcos teóricos y las metodologías propias de un campo en particular, avanzando en modos de trabajo que permitan quebrar la fragmentalidad heredada de la racionalidad hegemónica eurocéntrica que ha colonizado la producción de conocimientos en las universidades latinoamericanas, rechazando enfoques disciplinadores que han limitado la producción de conocimientos a ciertas temáticas o grupos de poder. Esto implica entonces comenzar a transitar un proceso de transformación encaminado a desarrollar formas novedosas y más democráticas de producir e intercambiar saberes, instituyendo nuevos espacios que logren consolidarse más allá de las fronteras que han demarcado las funciones tradicionales de las universidades y que apuesten a la incorporación de nuevos sujetos y nuevas formas de relación entre estos con la producción de conocimientos, los modos de aprendizaje y las maneras de vincularse con la sociedad.

Visto de este modo, entonces las clases no deberían centrarse en la transmisión de un saber acabado e indiscutible que es imperioso memorizar para ser reproducido y acreditado en el momento del examen; todo lo contrario, a partir del reconocimiento de la provisionalidad del conocimiento científico 
URL: https://www.revistas.una.ac.cr/index.php/dialogo/index

CORREO ELECTRÓNICO: universidadendialogo@una.ac.cr

DOI: https://doi.org/10.15359/udre.10-2.5

y de su subjetividad, debería fomentarse una construcción colectiva de un tipo de conocimiento áulico que recorra en su desarrollo tanto las trayectorias disciplinares como las experiencias históricas y metodológicas, y en especial los aportes de los estudiantes que, como sujetos activos, contribuyen y modulan la dinámica del aula, resignificando en ese proceso tanto el capital simbólico (Bourdieu y Passeron, 2009) heredado como aquel que han logrado "acumular" como parte de sus trayectos formativos y de un aprendizaje intervenido por las experiencias y los aportes de sus propios compañeros de clase.

La docencia no debería ser entonces un modo de perpetuar las tradiciones disciplinares, sino que bajo una perspectiva integradora sería posible posicionarse en una didáctica particular, propia de la disciplina que se enseña, y desde ella intentar reconstruir las lógicas que vertebran la producción de conocimientos tanto desde un método hipotético deductivo, como es usual en las ciencias naturales, como desde la hermenéutica, como eje vertebrador de la búsqueda de explicaciones en las ciencias sociales. En esta manera de abordar la enseñanza se enseña también un modo particular de cuestionar la realidad, reconociendo que los contenidos enseñados no se restringen a aquellos llamados conceptuales, sino también a la lógica propia de aquellas metodologías que permiten el acceso a este conocimiento.

De esta manera, se habilita la democratización de la palabra como punto de partida de una decisión pedagógica de facilitar la articulación del conocimiento con la realidad, y en esa lógica, las paredes físicas del aula se desmoronan y se extienden a la realidad de los barrios que se transmuta en nuevos y complejos escenarios de aprendizaje. En estos contextos, los saberes y contenidos que delimitan el programa de estudios de una materia debe ser entendido como un saber susceptible de ser interpelado, en diálogo, con otros modos de conocimiento, y en especial con el conocimiento experiencial, incluso sobre temas conceptuales de una disciplina en particular, que son resguardados por las tradiciones orales, las costumbres y las experiencias cotidianas de la comunidad.

Esta interacción de saberes no solo se limita al conocimiento en sí mismo sobre un objeto de estudio, sino que se constituye en una relación entre sujetos, y en esa relación es posible la construcción de saberes emergentes y la consolidación de un modo de revalorizar el conocimiento mismo a partir de la construcción de diagnósticos participativos sobre una realidad que, puesta en debate desde diferentes prismas, promueve el aprendizaje colectivo, la planificación de estrategias organizativas de la comunidad y la posibilidad de transformación social. 
ReVista Universidad en DiÁlogo • Vol. 10, N. 2, Julio-Diciembre, 2020 • 97-124

ISSN 2215-2849 • EISSN: 2215-4752

URL: https://www.revistas.una.ac.cr/index.php/dialogo/index Correo eleCtrónico: universidadendialogo@una.ac.cr

DOI: https://doi.org/10.15359/udre.10-2.5

En este proceso, la extensión como función explícita es integrada pedagógicamente al proceso de enseñanza-aprendizaje con un impacto extraordinario en la formación de los estudiantes en relación con el corpus de conocimientos de la materia bajo la que estos saberes se ponen en diálogo, pero sobretodo impacta por su relevancia en la formación de futuros egresados comprometidos con la realidad y en especial por la articulación de la universidad con la comunidad, que es entendida como un actor más de la sociedad en que se inserta.

No obstante, es fundamental distinguir esta mirada sobre el protagonismo de la universidad en relación con las demandas concretas de la sociedad, desde la perspectiva holística a la que se refiere Frondizi (1971) cuando la acota a la formación de profesionales con conciencia social y a la resolución de los problemas que afligen al país, un rol que, si bien denota una impronta progresista y desarrollista, no deja de ser sumamente ambiguo y generalista. El formato de integración entonces no puede, ni debe, restringirse a la transferencia, ya que la democratización de la palabra anula la posibilidad de que en la interacción entre sujetos prime un tipo de conocimiento sobre otro. Más aún, bajo este proceso subyace una lógica que incluso legitima la posibilidad de trabajar con base en hipótesis de acción que, si bien no intentan contrastar posiciones antagónicas sobre un tema en particular, operan de acuerdo al supuesto de que el valor emancipador del conocimiento permite transformar no solo a los sujetos que participan de esa interacción, sino también a la realidad en la que viven.

Finalmente, es interesante destacar el modo en que este formato de prácticas integrales impulsa nuevas preguntas derivadas de los diagnósticos participativos, de la observación e incluso de los debates emergentes en torno a las problemáticas territoriales, de tal manera que es el enfoque crítico el que favorece la evaluación permanente de los modos en que se objetiva la experiencia. Esta reflexión sobre la práctica genera nuevas preguntas de intervención que podrían suscitar otras preguntas de investigación o hipótesis, a partir de las cuales se puedan delinear nuevas líneas de trabajo, que bajo la lógica disciplinar terminan reformulando una investigación situada, que, además de exhaustiva y de excelencia (como criterio para ser legitimada por la comunidad académica), también resulte relevante y pertinente por responder a demandas concretas de la comunidad, y no a temas instalados por la agenda de los países centrales. Esto además de ser fundamental para el desarrollo local o regional, entendido bajo la premisa del bienestar colectivo y sobretodo de las clases populares, fomenta en países como el nuestro, y otros tantos de la región -considerados como periféricos en temas científicos-, un proceso de decolonización (Castro, 2007) de las prácticas académicas que 
URL: https://www.revistas.una.ac.cr/index.php/dialogo/index

CORREO ELECTRÓNICO: universidadendialogo@una.ac.cr

DOI: https://doi.org/10.15359/udre.10-2.5

permite avanzar en una agenda propia no solo en relación con la producción de conocimientos, sino también de la cultura académica que es constituida y reconfigurada permanentemente durante este proceso.

Como colofón de esta relación integradora en la búsqueda participativa de respuestas a este tipo de preguntas complejas, se terminan conformando equipos de trabajo interclaustros, interdisciplinares e intersectoriales, que en tanto logren trascender la barrera de lo "inter", que inicialmente los constituye, les es posible desarrollar enfoques transdisciplinares en el abordaje de una realidad que es compleja en sí misma y en relación con los modos de abordaje. Este modo de producir conocimiento y sentido (por las particularidades de su propia génesis epistémica) impacta favorablemente en el modo de objetivar la enseñanza en el aula y, en consecuencia, en el diseño de los programas de estudio que, además de incluir temas generales de la historia de la disciplina, sean capaces de incorporar otros tipos de problemas contextualizados, que den cuenta de un conocimiento comprometido, producido y legitimado por sujetos integrales en su modo de concebir y de reflexionar sobre sus prácticas.

Bajo este formato, toma verdadero significado el término intervención, en lugar de extensión, en su acepción histórica, transferencista y disociada de otras prácticas, lo cual se constituye en una estrategia de transformación de la realidad preexistente y en una manera instituyente (Kaplún, 2016) y profundamente reflexiva de habitar la universidad.

\section{Reflexiones finales...}

Como reflexión final, es posible acordar con la idea de que la extensión universitaria constituye una actividad compleja y polisémica, en su génesis y en su praxis, atravesada por posiciones pedagógicas e ideológicas claras, que incluyen, por los modos en que acontece y se objetiva, tanto la transferencia de conocimientos como la construcción dialógica y crítica de saberes de diferente naturaleza. Esta diversidad de formatos en los que se objetiva la extensión como práctica académica abreva en dos paradigmas epistemológicamente antagónicos, el transferencista, que implica un apego a la tradición original de la extensión en su versión semántica más genuina, pero que no propone un diálogo de saberes, sino la imposición de uno sobre otro (Kaplún, 2005), de modo tal que sus prácticas terminan, según Freire (1973), por ser una modalidad de invasión cultural, y otros formatos que bajo el paradigma crítico o problematizador, respecto de la realidad entendida en toda su complejidad, plantean una construcción colectiva de saberes bajo formas dialógicas de comunicación. 
Si bien los formatos en los que se explicitan han estado condicionados por las coyunturas históricas, las tradiciones disciplinares y la hibris propia de la interculturalidad, que confluye en el encuentro entre la academia y la comunidad, algunas de estas modalidades, entendidas bajo esta taxonomía como formatos, hoy son explícitamente cuestionadas bajo el término de extensión, en tanto otros comienzan a protagonizar un proceso instituyente en que la apuesta a la integralidad de las prácticas con una visión profundamente crítica e ideológicamente comprometida contribuye a desdibujar sus límites, anunciando el inicio del fin de una metáfora que disocia las prácticas universitarias atadas a los diversos modos de habitar la universidad.

En este sentido, la praxis reflexiva propia de un proceso que se retroalimenta en la relación objeto-sujeto fomenta un modo de entender el conocimiento como una confluencia de saberes que provienen de preguntas de investigación o de tradiciones orales o experiencias, y que a su vez pueden ser sometidas a contrastación, o pensadas como hipótesis de acción capaces de orientar la transformación social.

En esa conjunción, la docencia se revaloriza, resignificándose los contenidos curriculares, interpretando al aula como un sistema complejo donde se aglutinan trayectos formativos, trayectorias e historias de vida y objetivos institucionales, con propósitos explícitos e imaginarios respecto del tipo de profesional que se pretende formar y del rol social que deberían desempeñar hoy las universidades, donde el eje debería correrse del rol de educar para centrarse en el de formar sujetos críticos, reflexivos y comprometidos con su entorno y con el de los demás. Bajo este paradigma crítico, y en el marco del formato de la integralidad, la universidad debería asumir como misión FORMAR sujetos integrales capaces de transFORMAR la realidad.

\section{Referencias bibliográficas}

Baptista, P. y Kuenzer, A. (1999). Universidade - nucleos tematicos: em busca da indissociabilidade ensino-pesquisa-extensao. Educar em Revista., $\mathrm{N}^{\circ}$. 15: 1-4. https://bit.ly/2NIeAfp

Barraza, A. (2010). Elaboración de propuestas de intervención educativa. Universidad Pedagógica de Durango, México., 124 pp.

Bauman, Z. (2004). Modernidad líquida ( $3^{\text {ra }}$ ed.). Fondo de Cultura Económica de Argentina, S.A., Grafinor, S.A., Bs. As., Argentina, 232 pp. 
Becher, T. (1993). Las disciplinas y la identidad de los académicos. Pensamiento Universitario Año $1, \mathrm{~N}^{\circ} 1$.

Bourdieu, P. (1987). Los tres estados del capital cultural. Sociológica, 2 (5), 11-17.

Bourdieu, P. (2008). Homo Academicus. Buenos Aires: Siglo XXI, 320 pp.

Bourdieu, P. y Passeron, J. C. (2009). Los herederos: los estudiantes y la cultura. Argentina: Editorial Siglo XXI, 216 pp. https://bit.ly/2TKSwEz

Castro, S. (2007). Decolonizar la universidad. La hybris del punto cero y el diálogo de saberes. El giro decolonial. Reflexiones para una diversidad epistémica más allá del capitalismo global. https://bit.ly/2TGxO8Q

Ceccon, E. (2008). La revolución verde tragedia en dos actos. Ciencias, Vol. 1 (91), 21-29. https://bit.ly/3ay1Q4T

Chevallard, Y. (1998). La transposición didáctica: del saber sabio al saber enseñado. Argentina: AIQUE Grupo Editor. https://bit.ly/37cRmpl

Crisci, J. V. y Katinas, L. (2017). Las colecciones de historia natural: memoria colectiva de la humanidad. Museo, 29, 23-30. https://bit.ly/2RyEV0u

Edelstein, G. (1996). Lo metodológico. Un capítulo pendiente en el debate didáctico. En Corrientes didácticas contemporáneas. Buenos Aires: Editorial Paidós.

Fals, O. (2009). Elementos ideológicos en el Frente Unido de Camilo Torres: ayer y hoy. En L. Sablich (ed.), Una sociología sentipensante para América Latina (pp. 419-430). Colección Pensamiento Crítico Latinoamericano. Biblioteca Universitaria Ciencias Sociales y Humanidades. Argentina, Buenos Aires: CLACSO.

Flores, M. (2004). Implicaciones de los paradigmas de investigación en la práctica educativa. Revista Digital Universitaria, 5 (1), 2-9.

Freire, P. (1970). Pedagogía del oprimido. Montevideo, Uruguay: Editorial Tierra Nueva.

Freire, P. (1973). ¿Extensión o comunicación? La concientización en el medio rural. Buenos Aires, Argentina: Editorial Siglo XXI, 109 pp.

Fresán, M. (2004). La extensión universitaria y la universidad pública. Reencuentro, núm. 39, 47-54. https://bit.ly/2R9x5LE 
Frondizi, R. (1971). La universidad en un mundo de tensiones. Misión de la universidad en América Latina. Buenos Aires: Editorial Paidós. https:// bit.ly/2G8Txyd

Gezmet, S. (2014). La vinculación universidad-sociedad. Modelos de extensión y características de las interacciones. Compendio Bibliográfico, 23. https://bit.ly/2TFx3Nd

González de la Fe, T. (2009). El modelo de triple hélice de relaciones universidad, industria y Gobierno: un análisis crítico. ARBOR Ciencia, Pensamiento y Cultura, CLXXXV N. ${ }^{\circ}$ 738, 739-755. doi: 10.3989/ arbor.2009.738n1049

González, G. R. y González, M. (2003). Extensión universitaria: principales tendencias en su evolución y desarrollo. Revista Cubana de Educación Superior XXIII (1), 15-26. https://bit.ly/37e8wCS

Jeria, L. M. (2013). La relación educativa en el aula universitaria: una mirada desde los conflictos suscitados en ella. Revista Psicología Universidad Viña del Mar, 2 (4), 8-32.

Klimovsky, G. (1971). Estructura y validez de las teorías científicas. En D. Ziziemsky (ed.), Métodos de investigación en psicología y psicopatología (pp. 152-176). Buenos Aires: Editorial Nueva Visión.

Kaplún, G. (2005). Indisciplinar la universidad. En C. Walsh(ed.), Pensamiento crítico y matriz colonial: reflexiones latinoamericanas (213-250). UASB-Abya Yala: Quito. https://bit.ly/38qdbBX

Kaplún, G. (2016). La integralidad como movimiento instituyente en la universidad. InterCambios. Dilemas y Transiciones de la Educación Superior, 1 (1), 44-51. https://bit.ly/2TJCYRl

Kuhn, T. (1962). The structure of scientific revolutions. Chicago: Chicago University.

Laclau, E. y Mouffe, Ch. (2011). Hegemonía y estrategia socialista. Hacia una radicalización de la democracia. Buenos Aires: Fondo de Cultura Económica. https://bit.ly/37emDbg 
Langer, A. (2010). Investigación, transferencia, extensión y docencia: análisis de las concepciones en la política científica y universitaria de la Argentina. https://bit.ly/2sMXCVI

Leff, E. (2007). Complejidad, racionalidad ambiental y diálogo de saberes: hacia una pedagogía ambiental. Desenvolvimento e Meio Ambiente, $\mathrm{N}^{\circ}$ 16, 11-19, Editora UFPR. https://bit.ly/3anla4F

Moreno, T. (2011). Frankestein evaluador. Revista de la Educación Superior, XL (4), 119-131.

Ortega, F. (2011). Docencia y evasión del conocimiento. En F. Ortega (ed.), Ingreso a la universidad: relación con el conocimiento y construcción de subjetividades (pp. 89-106). Córdoba, Argentina: Ferreira Editor. https://bit.ly/36aLlrX

Regidor, J. G. (1987). Innovación tecnológica en la agricultura y acumulación de capital: un análisis crítico de la revolución verde. Revista Estudios Agro-Sociales, 142, 7-30. https://bit.ly/38jtdxo

Sánchez, N. E. (2012). Modelo actual de desarrollo agrícola de la Argentina. En A. Carrasco, y L. Tamagno (eds.), Modelo agrícola e impacto socioambiental en la Argentina: monocultivo y agronegocios (pp. 7-43). Serie Monográfica Sociedad y Ambiente.

Serna, G. A. (2007). Misión social y modelos de extensión universitaria: del entusiasmo al desdén. Revista Iberoamericana de Educación, $\mathrm{N}^{\mathrm{o}} 43$, 1-7. https://bit.ly/2NGfmJG

Sousa, B. (2007). La universidad en el siglo XXI. Para una reforma democrática y emancipadora de la universidad (4 ${ }^{\text {ta }}$ ed.). Colección Universidad. La Paz, Bolivia: Plural. 117 pp.

Tommasino, H. y Cano, A. (2016). Modelos de extensión universitaria en las universidades latinoamericanas en el siglo XXI: tendencias y controversias. Universidades, $\mathrm{N}^{\circ}$. 67, 7-24. https://bit.ly/2RzBKFF 
Tünnermann, C. (2001). El nuevo concepto de extensión universitaria. En

Memorias del V Congreso Iberoamericano de Extensión. Sociedad, Educación Superior y Extensión: balance y perspectivas. Ciudad de México, DF: Editorial ANUIES. https://bit.ly/2RyGc7M

Vaccarezza, L. (2015). Apropiación social e hibridación de conocimientos en los procesos de extensión universitaria. Cuestiones de Sociología, $\mathrm{N}^{\circ}$ 12. https://bit.ly/2TGHMah

Watson, J. B. (1913). Psychology as the behaviorist views it. Psychological Review, 20 (2), 158. https://bit.ly/2GaLCAB

Zavaro, C. (2019). Las prácticas de extensión como experiencias de curricularización: entre el debate y la práctica objetivada. Redes de Extensión, (5), 7-22. https://bit.ly/30DQhEs 